\title{
Development of a Home Energy Management System in Smart Grids: A Laboratory Test-bed and a Mobile Application
}

\author{
Imane L'hadi, Mohammed Bakr Sikal, Sarah Lahtani, Ahmed Khallaayoun, Rachid Lghoul, \\ Mouad Sahil \\ School of Science and Engineering \\ Al Akhawayn University in Ifrane \\ Ifrane, Morocco
}

\begin{abstract}
The current electricity grid in Morocco is facing challenges due to its conventional nature. The conventional sources of energy used are one of the major participants in pollution, and they are inefficient and unreliable in securing the national energy security. Morocco is energy dependent as it imports about $97 \%$ of its energy needs. To improve the current situation, the energy in the residential sector needs to be managed in order to reduce peak demand, protect the environment, reduce cost, avoid energy deficit, involve consumer, and efficiently use its renewable energy potential. This paper tackles the building blocks of smart grids, namely, the residential microgrid. The paper focuses on developing a home energy management system using a laboratory test-bed and a mobile application on smart devices (phones, tablets, etc.) in order to establish a two-way flow of power and data, and manage residual energy. The design, architecture and implementation of the mobile application and the test-bed are detailed. Test-bed hardware components are described. Methods for testing the application are suggested, and finally, algorithms for home energy demand optimization are presented as an extension of the home energy management mobile application features.
\end{abstract}

\section{Introduction}

As a developing country, Morocco has known a remarkable increase in energy demand and electricity consumption. According to [1], [2] and [3], from 2002 to 2011, the energy annual consumption has increased with an average rate of $5.7 \%$ with a record of 85.9 billion dirhams (MAD) in Morocco's gross energy bill for 2011 due to an energy consumption of 200 trillion Wh. Electricity demand has also increased in the past decade at an average rate of $7 \%$ until it has reach 28.8 trillion Wh [3], [4]. Morocco is considered the largest energy importer in North Africa with an energy dependency rate on foreign imports of $95.6 \%$ recorded in 2011 [2]. Still, the majority of the energy sources are conventional where petroleum products account for $62.9 \%$ of the energy consumption, $22.5 \%$ is covered by coal, and the remaining is covered by electricity trade (imports minus exports), natural gas and renewable energy sources (RES) [1], [4]. One of the major negative impacts of the stated energy consumption distribution is environment pollution. The current electricity grid in Morocco is not energy efficient as it is a unidirectional grid. Only one third of fuel energy is converted into electricity knowing that the waste heat is generally not recovered. In addition, during transmission, $8 \%$ of the grid output is wasted, and $20 \%$ of its generation capacity is used to meet peak demand only [5]. Morocco, however, has a gap of improvement, which is RES. In [4] and [6], Morocco is identified to have both the potential to be a regional hub that ensures the interconnection between Europe and North Africa, and the potential to be a major renewable energy producer. According to [4] and [7], Morocco registers an average of 2300 $\mathrm{KWh} / \mathrm{m} 2$ per year of solar radiance along with an immense potential for wind power found especially in the north and the south. Therefore, the question is, what does Morocco need to do in order to overcome these weaknesses and cease the available opportunities?

In the past decades, the smart grid has seen a significant interest due to environment issues, increasing fuel cost and increasing energy demand. Morocco needs to keep up with the demand, maintain the same quality, and introduce management at the demand side by changing the electricity grid into a smart one. The first step into achieving the mentioned goal consists of developing smart microgrids that constitute the building blocks of the larger smart grid. Microgrids at the residential level, for instance, need to be equipped with a management tool that consists of smart meters and an energy management system (EMS).

The latter has a set of predefined key requirements, which are monitoring, disaggregation, availability and accessibility, information integration, affordability, control, cyber-security and privacy, and finally, intelligence and analytics. The EMS should provide real-time information about the energy consumption. The energy consumption of individual appliances should be available. The system should also integrate other information such 
as historical data and energy usage of the different appliances. The consumer should be able to access information at any time through a user-friendly interface. In addition, the EMS should be easy to install, configure and maintain while consuming minimal energy and requiring low running cost. Most importantly, the system should ensure data and control signal security as well as the privacy of the consumers' consumption profiles. Since consumers have limited time and knowledge to make energyrelated decisions, it is necessary to have an intelligent system that is able to seamlessly come up with measures that will ensure energy saving for the home owner [8].

The remainder of the paper is structured as follows: Section 2 introduces the management tool components, and presents the suggested EMS. In Section 3, the mobile application architecture and design are detailed, while its implementation is presented in Section 4. Section 5 suggests different testing methods. Section 6 details the laboratory testbed architecture, components and workflow. Section 7 introduces algorithms for home energy demand optimization, and finally, Section 8 concludes the paper and Section 9 sheds the light on the future work.

\section{The Management Tool Components}

\subsection{Smart Meters}

As discussed earlier, fulfilling the two-way flow of data and energy is imperative in developing a residential microgrid. The latter is one of the major reasons for moving toward a smart grid and cannot be achieved without the presence of a smart meter. Thus, what is a smart meter? What does it do? Is it smart enough?

Smart meter can be defined as a digital power meter with improved communication capabilities to notify the consumers about their energy usage [9]. The smart meter is coupled with an in-home display (IHD) that informs the consumers about their wholehouse power and energy consumptions, but does not provide them with enough transparency in regards to their individual appliances consumption.

In [9], a demand response (DR) example scenario is provided to show the limitations of smart meters and conclude that smart meters are not actually smart. The scenario states that a house occupant receives on his IHD a DR sign through a smart meter to notify them that they have two minutes to reduce their consumption by $5 \mathrm{KW}$ if they want to receive a financial incentive. The occupant checks first the IHD and finds out a current demand of $15 \mathrm{KW}$. The challenge for the occupant is to find out which appliances are consuming the $5 \mathrm{KW}$ that they need to reduce. A trial and error method might be useful if the occupant turns off each appliance one at a time, and checks the displayed demand in the IHD. However, the latter is not practical within the twominute timeframe.

Thus, from the scenario, we can say that the smart meter did not help the occupant make a decision; it just facilitated the coordination of the occupant decision-making process. The key component, therefore, that we are looking for is a meter with the intelligence that can provide the occupant with potential appliances to switch off in order to cut the consumption by $5 \mathrm{KW}$. The necessary meter is a meter with a load disaggregation intelligence, which is the cognitive smart meter, or the cognitive power meter.

A cognitive meter is defined as a smart meter with embedded load disaggregation intelligence [9]. In other words, a cognitive meter is a meter that informs consumers not only about their whole-house power and energy consumption, but it also enables them to monitor every single load at the consumer side. In the scope of this paper, load disaggregation is done using the cognitive meter that plays the role of a gateway. The latter receives data about energy consumption from either smart appliances or from sensors installed in appliances. It is part of the proposed energy management system.

\subsection{Energy Management System: State of the Art}

There are several EMS that fulfill some of the predefined key requirements, but fail to provide others. Thus, the available EMSs do have different features, but also certain limitations [8]. PERSON is an EMS, and it is based on the Pervasive ServiceOriented Networks. It is known for its low cost and low energy consumptions, and it offers mainly monitoring and controlling tools [8]. However, PERSON lacks privacy and security. WattDepot is an open source service-oriented framework for energy management, but it does not have any programmable or automatic control and its lacks intelligence [8]. ViridiScope is an EMS designed for "Fine-grained" monitoring of residential energy consumption. It is affordable and ensures information integration. However, its does provide neither security nor manual, programmable or intelligent appliances control [8].

Mobile-Based Feedback System is a mobile phone application that is developed to monitor and measure energy consumption of every appliance using a smart meter. In this EMS, operations are not automated and security along with remote control is not provided [8]. DEHEMS is a digital home EMS that provides feedback to consumers based on the collected user experiences and preferences. One of the DEHEMS' limitations is the lack of security and privacy as well as control of appliances [8]. There is another energy management mobile application 
named EnergyWiz that provides energy consumption information and compares it with other consumers to change practices and correct behavior, but it also lacks privacy and security, appliances control, intelligence, automation of operations, and information integration [8]. NOBEL is also an EMS, but user-dependent. It supports decisions using data collected from the consumer. Also, no privacy and information integration provided [8]. Last but not least, ALIS is an Adaptive Living Interface System for monitoring, controlling and feedback. The main limitations of ALIS are in terms of affordability, intelligence, security and privacy [8].

\subsection{What makes our EMS different?}

Relying on the fact that, in 2012, 15.6 million Moroccan individuals aged between 6 and 74 are connected to Internet while $56 \%$ of these connected people use Internet on a daily basis [10], and inspired from the existing EMS, the proposed energy management system is a mobile application that encapsulates the predefined key requirements, and overcomes the encountered limitations. The latter is the first ring from the smart grid chain that emphasizes on peak shaving and energy saving. In other words, the proposed EMS will enable the adequate management of electricity when there is a huge demand, which will help in reducing the cost, the budget, and avoid deficit. It will enable the consumer to be conscious of his or her energy consumption. In addition, a new feature is introduced, which is the production of renewable energies. The consumer will be able to produce energy and use it while targeting an optimized load profile. The latter will help in minimizing the overall energy demand at the national level, and reducing the pollution caused by the use of conventional energy sources. The EMS will also provide the consumer with information about individual appliances and sources through a user-friendly interface that will enable monitoring and controlling at a low running cost and energy consumption. The system is designed to propose and make decisions while ensuring security and privacy of the user.

To summarize, the suggested mobile application is a tool to reduce the consumer's electricity bill, to protect the environment through the use of RES, to involve the consumer as a producer of renewable energy, and to ensure consumption and production transparency in order to avoid any surprises in the electricity bill. The architecture of the suggested EMS is detailed in the next section.

\section{EMS Architecture}

The purpose behind establishing the system architecture is to draw a global picture of the system, and build the connectivity between the physical layer and the information system layer. The role of the presented architecture is to provide a view of the system from different angles, and to show the flow of data, instructions, and decisions from one layer to the other. Thus, in this section, the EMS architecture is presented in Fig. 1, and a detailed explanation and a sketching of every layer are provided.

Fig. 1 shows the different components of every layer in the architecture. Within a house that is connected to the smart grid, the owner has appliances and RES that he or she wants to control and monitor using a mobile application loaded on his or her smart device (smartphone, tablet, etc.). Once the user accesses the application, a communication between the client side and the server side is established through Hypertext Transfer Protocol (Http). Any reading or writing from and in the application is done from and in the database at the level of the information system layer.

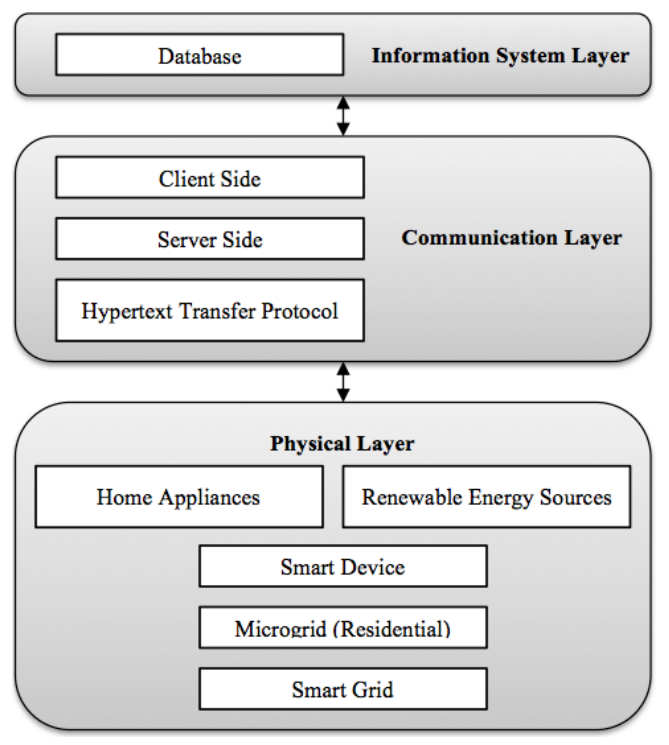

Figure 1. EMS Architecture

\subsection{Physical Layer}

The physical layer is basically a house model that demonstrates the connection between the appliances, the RES, the smart grid and the smart device (mobile application). The house contains copious appliances such as light bulbs, heater, washing machine, TV and fridge along with a renewable energy source, which is in our case a PV system. The RES and the smart grid feed in the house with energy, while the RES might also inject into the grid. A gateway is inserted between the house and the smart device in order to ensure communication. The latter is split into two stages. The first stage is the home appliances/sources and the gateway communication established using Zigbee Protocol. The second stage is the communication between the gateway and the mobile application ensured using Wi-Fi. Finally, the 
gateway can also telecommunicate with the smart grid via all sorts of technologies (Ethernet, Wi-Max, $\mathrm{Wi}-\mathrm{Fi}$, etc.) but for the time being, in the architecture we suggest, the database could be seen as the smart grid as that is were all the decisions are made. Fig. 2 is a detailed representation of the physical layer for better understanding of the communication between the different entities.

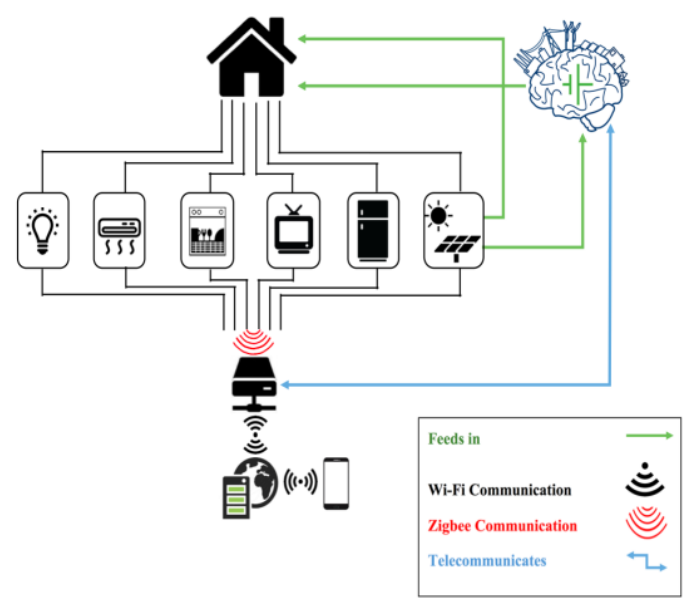

Figure 2. Physical Layer

\subsection{Communication Layer}

The communication layer is the intermediary layer between the physical layer and the information system layer in which the communication between the client side and the server side is established through Http. As shown in Fig. 3, this layer is the set of events that happens when the user opens the application. First, the application sends an Http request to the server. The latter acknowledges the request, and replies with the requested page's source code. When the application receives the source code, it renders it to a viewable content and displays it to the user. Note that the application is a web application that is loaded through a web view in a mobile application. For example, if the user wants to view the past consumption of an appliance, in the application, an appliance menu is displayed from which the user can select "Past Consumption". The application sends a GET Http request to the server. The server executes the Hypertext preprocessor (Php) script, which sends a Structured Query Language (SQL) query to retrieve data from the database. The server returns a Hypertext Markup Language (HTML) page containing the retrieved data to the application. Afterward, the application renders the code to a viewable content, and displays the requested past consumption.

\subsection{Information System Layer}

The information system layer consists of a database that enables the display of the requested information, and the storage of the entered information from and in the mobile application. The database is composed of classes, attributes and relationships shown in Fig. 5. The presented entityrelationship diagram (ERD) is a summary of the conducted software engineering study in which different diagrams were used (the sequence diagrams, the class diagram and the use case diagram) in order to come up with a complete database design. It models a house with rooms, smart-plugs, appliances and sources. Each appliance has consumption history, and each source has production history. The database also contains pieces of information and advice the user might be seeking.

When a new appliance is purchased and added in the application, it is stored along with its attributes in the appliance's table. The latter is identified by the smart-plug to which it is connected. The plug is located in a specific room in the user's house. The history of appliances' consumption and the history of sources' production are stored into the database regularly with a granularity chosen by the user.

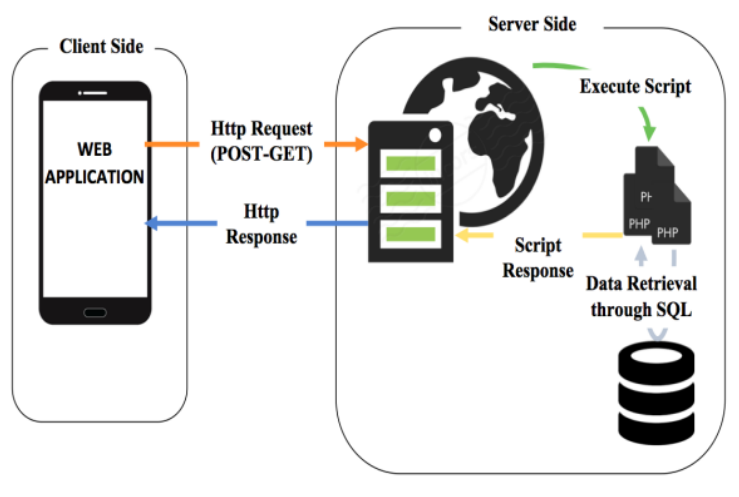

Figure 3. Information System Layer

\section{EMS Implementation}

Initially, the EMS mobile application was implemented as an Android mobile application. One of the limitations of this approach was the limited use of this application by only Android devices' owners, and trying to develop an iOS version would be a redundant work and that would require mastering its native programming language. Let alone, if we consider developing also a Blackberry version, and a Windows version. Therefore, we opted for a responsive web application that can adapt with all screen sizes. The user can, then, access the application from any mobile device, and even from a web browser. To upload the application on an Android device, we simply created an Android application in which web viewing is enabled. 


\section{EMS Testing Methods}

Once done with the implementation phase, testing comes after as a necessary step to check the alignment of the implemented functionalities with the design and the requirements. In order to test the developed EMS, three options are available. The first one is having a real house and an existing smart grid. However this method is still unfeasible since Morocco still does not have a smart grid, and the used appliances in households are neither smart appliances nor appliances equipped with sensors and relays to be able to receive data from them and control them.

The second one is building a model house in a database and populating it with all necessary data (consumption, peak-time tariffs, etc.). This is actually the method that was initially used to test the EMS. After implementing all the functionalities, a database is constructed and populated with necessary data about appliances, sources, consumption and production from excel files built from different forums, and websites of different brands of appliances and sources. Both the application and the database are hosted in a web server. The application is loaded once the user opens the web viewing android application, and he or she can read from the database or write in it depending on the selected functionality.

The last option is building a test-bed in the laboratory containing a house model that is connected to the application through a gateway.

\section{Laboratory Test-Bed}

\subsection{Test-Bed Architecture}

According to Fig. 4, the device to be monitored could either be a home appliance or a renewable energy source. The sensing and control layer consists of a current sensor to measure the device consumption, and a relay to control the device and set it on and off. Additional sensors (Temperature, Light) may be added accordingly. At the level of the smart plug, there is an Arduino Uno that collects data from the sensors, and sends it to the gateway via Zigbee using the Xbee connected. The smart plug also receives commands from the gateway to set on/off the appropriate device using the Control relay circuit. The gateway consists of an Arduino Uno, connected with an Xbee Shield and an Ethernet Shield. Its purpose is to collect data from the smart plugs and communicate it to the database via Ethernet, and also get commands from the database and send them to the smart plugs via Zigbee. On the other hand, the database collects data about the devices consumption and status (on/off). The web application is the EMS and the intermediary to help the end user to visualize data from the database and to send commands to the smart plugs.

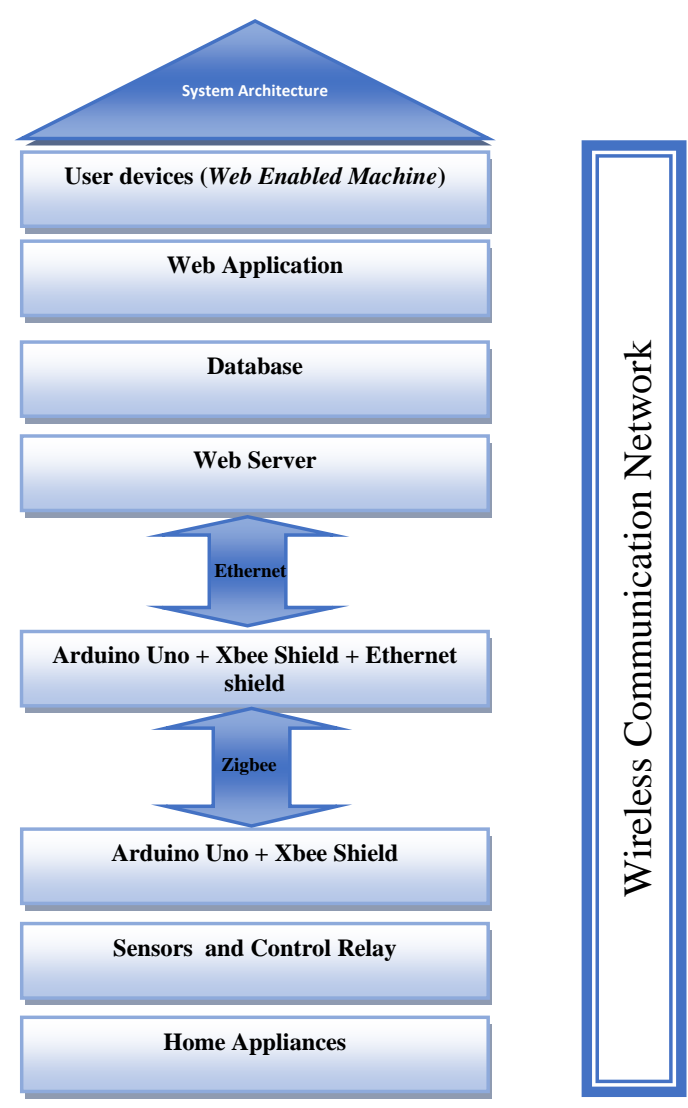

Figure 4. Information System Layer

\subsection{Test-Bed Components}

\section{- Smart Plugs}

- Arduino Uno: is an open source microcontroller development platform that uses the Atmega 328P microcontroller chip. In the proposed test-bed architecture, each home appliance and each renewable energy source is linked to a dedicated smart plug responsible for:

- Sending the sensed data to the gateway

- Executing the commands sent by the gateway

- Xbee radio: Xbee radio is a module used for wireless communication between microcontrollers, computers or any device with serial port to enable communication protocols like Zigbee. The chip is stacked over the Arduino Uno microcontroller and it is configured 
to be used as both a transmitter and a receiver:

- Transmitter Mode: this mode is needed to transmit the data sensed by the light, temperature and current sensors to the gateway.

- Receiver Mode: this mode allows the smart plugs to receive commands from the gateway. For instance, if the user chooses to switch-off the heater, the gateway retrieves its new status from the database and notifies the corresponding smart plug. The latter then applies a low voltage on the heater to turn it off.

- Temperature Sensor: The temperature sensor detects temperature in the form of resistance value using a thermistor. The Arduino Uno converts the voltage measured by the input pin to the real value of temperature in ${ }^{\circ} \mathrm{C}$ that varies between -40 to $125^{\circ} \mathrm{C}$, with an approximated error of $1.5^{\circ} \mathrm{C}$.

- Light Sensor: The light sensor uses a light-dependent photoresistor that detects light and which resistance decreases when light intensity increases.

- The Split-Core Current transformer sensor YHDC STC 013-030: the chosen sensor is a non-invasive current sensor that does not require the Arduino Uno to be connected to a monitor.

- The Gateway

- Arduino Yún: is an open source microcontroller development platform based on both ATmega32u4 and the Atheros AR9331. The built-in WiFi and Ethernet support allow the device to be constantly connected to the web server in order to:

- $\quad$ Read the updated statuses of every home appliance from the database

- Send the new updates to the corresponding smart plugs (using the Xbee radio)

- Write the sensed data received from the smart plugs to the database

- Xbee radio: this chip is stacked over the Arduino Yún in order to allow the gateway to communicate with the smart plugs. Both the transmitter and the receiver modes of operation are used in this module as well to enable the gateway to perform the tasks it is responsible for.

\subsection{Test-Bed Workflow}

In the proposed architecture as shown in Fig. 6, every home appliance is connected to a smart plug, which consists of an Arduino UNO, a relay box, a current sensor, and a light sensor. Each smart plug collects data from the sensors and sends it to the gateway via ZigBee, which writes it to the database via Wi-Fi. The application allows, through the "Monitoring module", the user to consult these data. The "Control Module" can also be tested with this architecture as the gateway is constantly checking the status of the appliances and sending them to the corresponding smart plugs. After the user switches on or off an appliance through the application, its status is changed at the level of the database. The gateway detects the modification by sending HTTP requests to the database server, and sends a message to the corresponding smart plug, via Zigbee, notifying it with the new appliance's status. The latter applies a high or low voltage on the appliance causing it to switch on or off accordingly.

\section{Toward an optimal EMS: Algorithms for Home Energy Demand Optimization}

Once the smart grid replaces the conventional grid, the human dimension becomes one of the major influencers of the grid efficiency, especially at the microgrid level (residential). In other words, residential users are seen as actors who play an essential role in improving the network efficiency when adopting intelligent mechanisms for energy demand management [11]. In the smart grid, huge amount of data is made accessible for the users such as appliances power consumption [12], [13] and realtime information on the economic value of energy [11]. In addition, with the distinctive feature that the smart grid is offering which is the two-way flow of electricity and information between the grid and the customers [14], users can send to the grid the consumption data of each of their appliances, and these data can be used by the mechanisms for demand load management. These mechanisms guide users in the process of decision making about their energy demand profile with the purpose of, first, reducing the electricity bill, and second, using efficiently the energy (reducing wastes) [11]. The latter aims at introducing a revolution in the social approach to electric energy with the ultimate goals of both reducing the users' electricity bill and improving the performance of the overall energy system [11]. In order to exploit the opportunities presented through this revolution, it is necessary to use Information and Communication technologies (ICT) components along with the mechanisms of the grid. As in this paper an EMS is already developed to work as a user interface and a facilitator of the two- 
way flow of information and electricity, the features of the EMS could be extended to serve as a tool for home energy demand optimization. These additional features can be implemented through the use of optimization algorithms. Thus, the question to be asked is: what type of optimization algorithms would be more appropriate to satisfy this need?

The most efficient way to design the appropriate algorithm is to solve a scheduling problem [15]. Thus, over the years, several algorithms has appeared in the literature using different optimization techniques which are: linear programming (LP) [16], Particle Swarm Optimization (PSO) [17], Adaptive Dynamic Programming (ADP) [18], Fuzzy-Logic [19], and Ant Colony Optimization [20]. Since The LP algorithm has been proven to offer the best solution with very low complexity compared to the other algorithms [15], the focus in this section will be on LP algorithm.

In [11], the authors opt for Mixed Integer LP model applied in two scenarios. First scenario is a single-user scenario in which single users manage individually their electricity demand. Thus, the LP model is designed to schedule the energy plan of a single house for the next day. In the second scenario, users are expected to cooperate in the management of their energy demand. Therefore, the energy plan is scheduled for a set of users. Since the designed EMS in this paper is build upon the first scenario, the focus would be on the single-user model only. In the considered residential scenario, the house model is a house equipped with a set of appliances to be used during the day at a specific period depending on the users preferences, solar panels that produce energy, and batteries that enable the storage of energy. The objective function of this model is to minimize the daily energy bill while scheduling when to buy, sell and store energy, and when to power on or off the home appliances [11].

The authors in [11] used a Mixed Integer LP model as they used two types of variables. The first type of variables is binary variables to represent to state of the appliances (on/off), and the second type of variables is continuous non-negative variables to represent the amount of energy sold and bought. In order to define a time horizon, the 24-hour daytime was divided into 15 minutes time slots resulting of 96 periods represented by the set $\mathrm{T}$.

\subsection{Objective Function}

As mentioned earlier, the objective is to minimize the daily electricity bill. The latter can be calculated using the difference between the costs of the total amount of energy bought and the total amount of energy sold in every one of the 96 periods. Thus, the objective function can be summarized as follows [11]:

$$
\operatorname{Min} \Sigma \mathrm{t} \varepsilon \mathrm{T}(\mathrm{ct} \cdot \mathrm{yt}-\mathrm{gt} . \mathrm{zt})
$$

where $y_{t}$ and $z_{t}$ represent, respectively, the total amount of energy bought and sold, while $c_{t}$ and $g_{t}$ represent, respectively, the costs of bought and sold energy.

\subsection{Constraints}

- Activity scheduling constraints: Every activity of an appliance is associated with a start time and an end time. Thus, the constraint should ensure that an activity starts exclusively at one time slot and is carried over the specified time interval.

- Batteries constraints: The battery can experience three possible modes which are charging, discharging and off. Therefore, we need to ensure that the battery is in one of the modes one at a time. In addition, battery energy at a specific time period is dependent on the energy in the battery from the previous period. Hence, another constraint is needed to explicitly express the relationship.

- Balancing constraints: These constraints emphasize, first the balance between the consumed and the produced energy, and second, the limits imposed by the grid in terms of the maximum energy to buy from the grid. The latter should not exceed the Contractual Peak Power (CPP) limits.

In [11], the constraints equations are explained in details.

\section{Conclusion}

A home energy management system has been developed using a laboratory test-bed and a mobile application on smart devices to establish a two-way flow of power and data, and to ensure residual energy management. On of the main objective of the proposed EMS is to change the Moroccan electricity grid into a smart one in order to keep up with the demand, and benefit from the immense renewable energy potential. The path toward building a smart grid requires the establishment of microgrids. Among the latter, we can list the microgrids at the residential level, which need to be equipped with a management tool that consists of smart meters and an EMS. The test-bed and the mobile application have been described together with the different algorithms for Home Energy Demand Optimization.

\section{Future Work}

As far as future work is concerned, a test-bed for a smart home is being implemented at the smart grid group. The test-bed currently consists of smart plugs using an Arduino UNO for each appliance along with a PV system that includes batteries. 
Optimization algorithms are also being implemented as for a purpose to reduce the consumers' electric bill while minimizing the dependency on the grid.

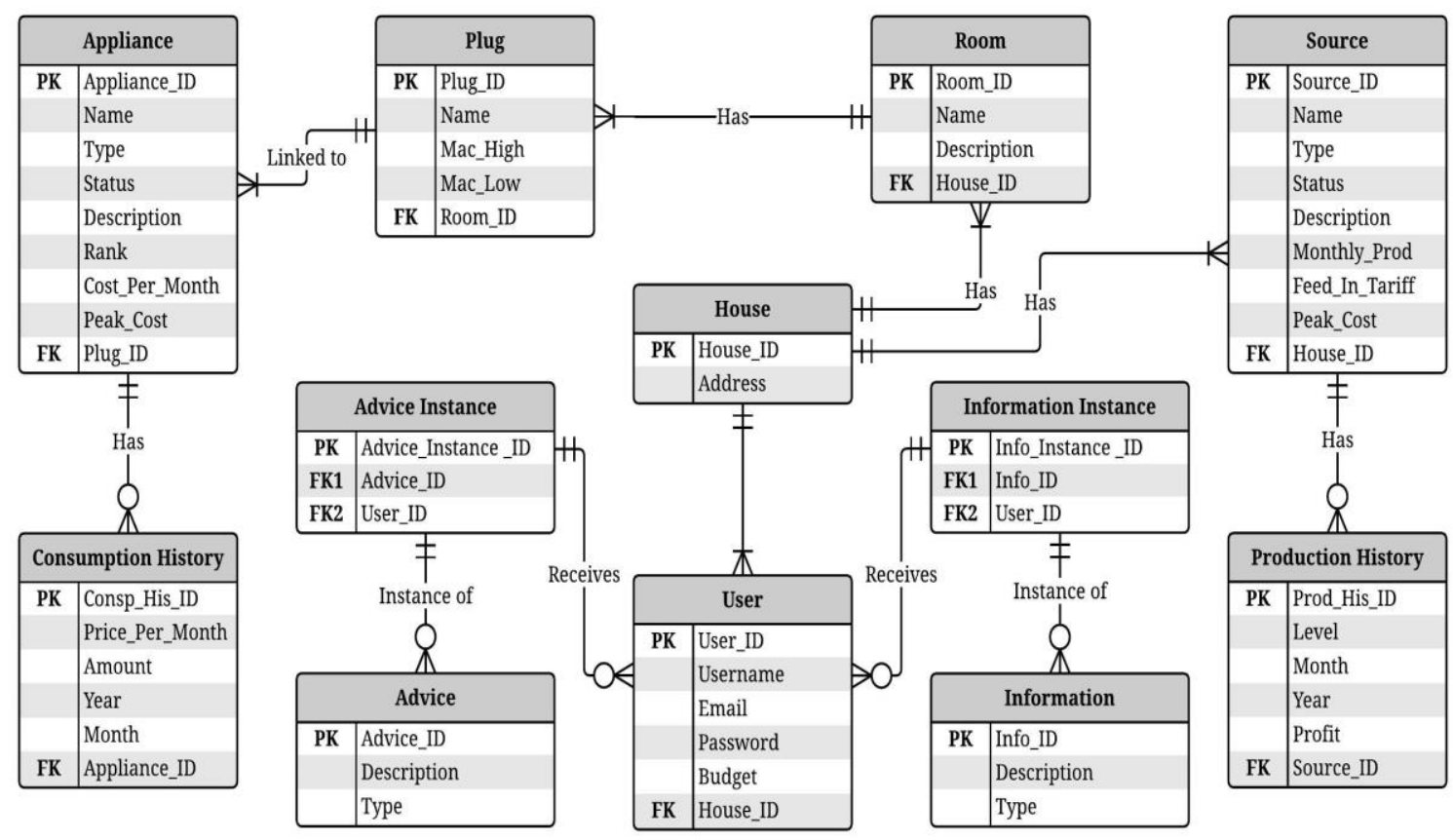

Figure 5. Information System Layer: Entity Relationship Diagram

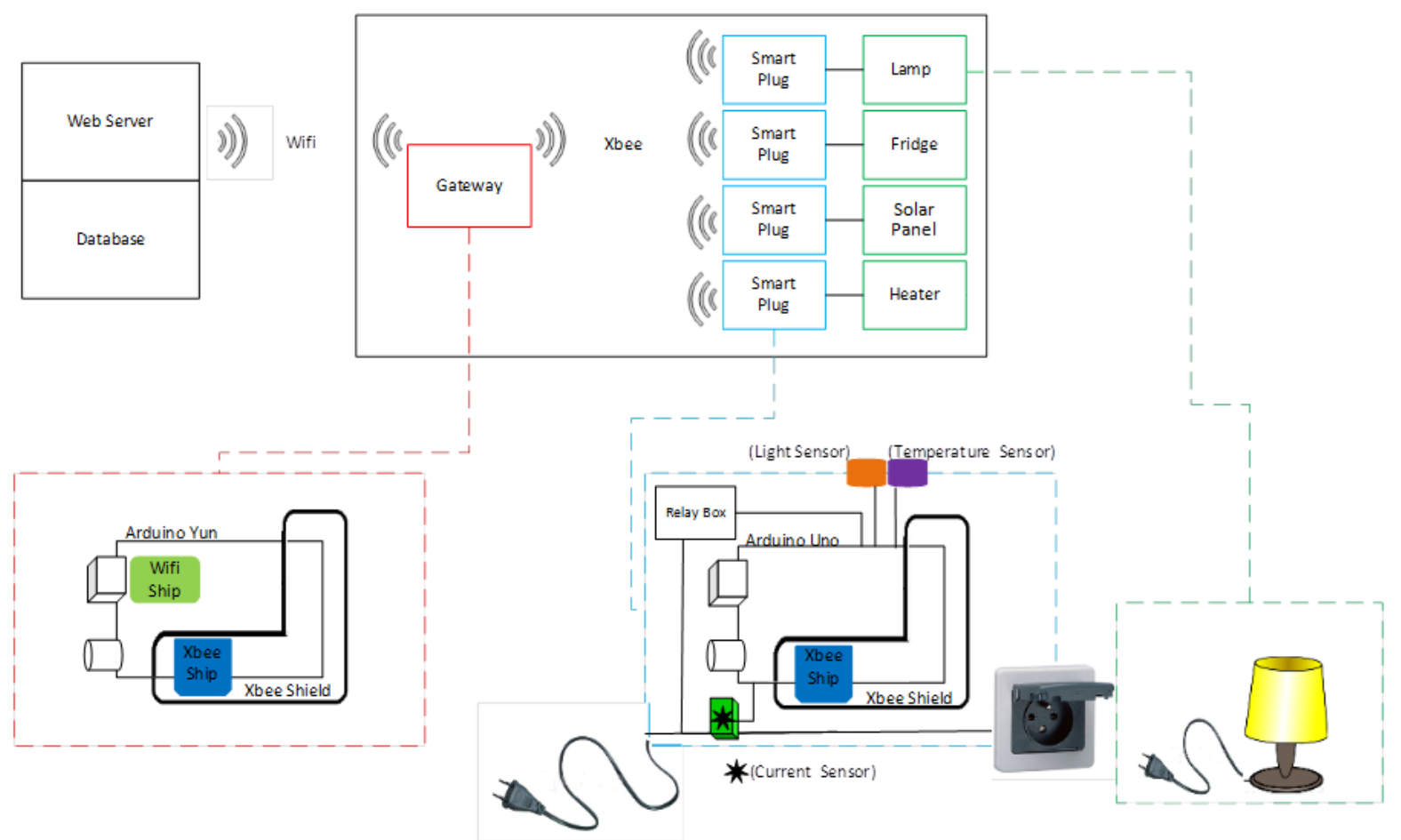

Figure 6. Laboratory Test-Bed Workflow 


\section{Acknowledgement}

This work was supported by $\mathrm{Al}$ Akhawayn University research grant number 92872 .

\section{References}

[1] MEMWE, "An overview of the energy sector in Morocco", Ministry of energy, Mines, Water and Environment, Morocco, 2013.

[2] G. Escribano-Frances, and E. S. M. Gonzalez, "Morocco, the European energy policy: an environmental approach", ed. F. Morata, 1.Solorio Sandoval, pp. 193-210, 2012.

[3] ONEE, "Rapport Annuel 2011”, Office National de L'Electrivité et de l'Eau Potable, Morocco, 2011.

[4] C. C. cîrlig, "Solar energy development in Morocco", Library Briefing: Library of the European Parliament, 2013.

[5] H. Farhangi, "The Path of the Smart Grid", IEEE Pwer \& Energy Magazine, Vol. 8, pp. 18-28, 2010.

[6] IRENA, "Renewable energy country profile: Morocco", International Renewable Energy Agency, European Union, 2010.

[7] ADEREE, "Programme d'efficacité énergétique", National Agency for the Development of Renewable Energy and Energy Efficiency, Morocco, 2009.

[8] S. Makonin, F. Popowich and B. Gill, The cognitive power meter: looking beyond the smart meter. In CCECE '26, pages 1-6. IEEE, 2013.

[9] S. Aman, Y.Simmhan and V.K. Prasanna, Energy management systems: state of the art and emerging trends. IEEE Communications Magazine, pp. 114$119,2013$.

[10] ARNT, "Etude sur l'usage des Technologies de l'Information et de Communication au Maroc", Agence Nationale de Réglementation des Télécomunications, Morocco, 2012.

[11] Barbarto, A. Capone, M. Delfani, M. Merlo, and A. Zaminga, House energy demand optimization in single and multi-user scenarios. InSmartGridComm '11, pp. 345-350, 2011.

[12] X. Jiang, S. Dawson-Haggerty, P. Dutta and D. Culler, Design and implementation of a high-fidelity AC metering network. In IPSN '09, pp. 253-264. IEEE, 2009.
[13] N. Bressan, L. Bazzaco, N. Bui, P. Casari, L. Vangelista and M. Zorzi, The Deployment of a Smart Monitoring System using Wireless Sensors and Actuators Networks. In SmartGridComm '10, pp. 49-54. IEEE, 2010.

[14] X. Fang, S. Misra, G. Xue, and D. Yang, Smart grid - the new and improved power grid: A survey. IEEE Communications Surveys and Tutorials (COMST), 14, pp. 944-980, 2012.

[15] S. Squartini, M. Boaro, F. De Angelis, D. Fuselli, and F. Piazza, Optimization algorithms for home energy resource scheduling in presence of data uncertainty. In ICICIP ‘4, pp. 323-328. IEEE, 2013.

[16] H. Morais, P. Kadar P. Faria, Z.A. Vale, and H. M. Khodr, Optimal scheduling of a renewable microgrid in an isolated load area using mixed-integer linear programming. Renewable Energy - An International Journal, Vol 35, issue 1, pages: 151156, 2009.

[17] N. Gudi, L. Wang, V. Devabhaktuni, and S.S.S.R.Depuru, A Demand-Side Management Simulation Platform Incorporating Optimal Management of Distributed Renewable Resources. Proceedings of Power Systems Conference and Exposition (PSCE), pp.1-7, 2011.

[18] D. Fuselli, F. De Angelis, M. Boaro, D. Liu, Q. Wei, S. Squartini, and F. Piazza, Optimal Battery Management with ADHDP in Smart Home Environments. Advances in Neural Networks - ISNN 2012, LNCS Springer, Vol 7368, 2012.

[19] R. H. Liang, and J. H. Liao, A FuzzyOptimization Approach for Generation Scheduling with Wind and Solar Energy Systems. IEEE Transactions on Power Systems, Vol 22, Issue 4, pp. 1665-1674, 2007.

[20] C.M. Colson, M.H. Nehrir, and C. Wang, Ant colony optimization for microgrid multi-objective power management. IEEE, Electrical and Computer Engineering Department, Montana State University, Bozeman, MT, USA, 2009. 